\section{Ventral Posterior Medial Nucleus}

John E. Mendoza

Department of Psychiatry and Neuroscience, Tulane Medical School and SE Louisiana Veterans Healthcare System, New Orleans, LA, USA

\section{Definition}

Specific somatosensory relay nucleus of the thalamus. The major input into this nucleus is via the ventral and dorsal trigeminothalamic tracts and the mesencephalic tract of V, which carry information regarding proprioception, both fine and crude touch, vibratory sensations, pain, and temperature from the face and forehead. In turn, the major cortical projection area for this nucleus is to the facial area of the primary somatosensory cortex (Brodmann's areas 3, 1, 2). 